\title{
THE LiCore POWER PLANT SIMULATOR OF THE MOLTEN SALT FAST REACTOR
}

\author{
Laureau A. ${ }^{1}$, Rosier E. ${ }^{2}$, Merle E. ${ }^{3}$, Beils S. ${ }^{2}$, Bruneau O. ${ }^{4}$, Blanchon J.C. ${ }^{4}$, Gathmann R. ${ }^{4}$, Heuer \\ D. ${ }^{3}$, Passelaigue . $^{3,4}$, Vaiana F. ${ }^{2}$, Zanini A. ${ }^{3,4}$ \\ ${ }^{1}$ Laboratory for Reactor Physics and Systems behaviour (LRS) \\ Ecole Polytechnique Fédérale de Lausanne (EPFL), CH-1015 Lausanne, Switzerland \\ ${ }^{2}$ Framatome \\ 10 rue Juliette Récamier, 69006 Lyon, France \\ ${ }^{3}$ LPSC-IN2P3-CNRS / Université Grenoble Alpes / Grenoble INP \\ LPSC 53 rue des Martyrs, 38026 Grenoble Cedex, France \\ ${ }^{4} \mathrm{CORYS}$ \\ 44 rue des Berges, 38024 Grenoble Cedex 01, France

\begin{abstract}
laureau.axel@gmail.com, emeline.rosier@framatome.com, merle@lpsc.in2p3.fr, stephane.beils@framatome.com, olivier.bruneau@corys.fr, jean-christophe.blanchon@corys.fr, ralf.gathmann@corys.fr, daniel.heuer@1psc.in2p3.fr, florian.passelaigue@phelma.grenoble-inp.fr, florian.vaiana@framatome.com, zaniniandrea@live.com
\end{abstract}

\begin{abstract}
Molten salt reactors as liquid-fuelled reactors are flexible in terms of operation or design choices, but they are very different in terms of design, operation and safety approach compared to solidfuelled reactors. Such reactors call for a new definition of their operating procedures and safety approach. Dedicated developments and studies have been performed in the frame of the European SAMOFAR project of Horizon2020 and in parallel in France involving CNRS, CORYS and Framatome to develop a system code called LiCore adapted to such reactors, corresponding to a basic-principle power plant simulator. The neutronic model LiCore, at the centre of the simulator, corresponds to an improved point-kinetics model to take into account the specificities of a MSR, notably the circulation of the delayed neutron precursors out of the core. Coupled to a simple piston model for the fuel motion in the core, this code can perform calculations faster than real time to simulate the behaviour of the fuel circuit. Transient calculations performed with LiCore are presented, together with comparisons first to a simple point-kinetics model and then to 3D calculations with the TFM-OpenFOAM coupled code. Finally, the LiCore code has recently been integrated in the ALICES platform, the integrated simulation toolset designed by CORYS for the development, maintenance and operation of major simulator such as power plant simulators.
\end{abstract}

KEYWORDS: Molten salt reactors, power plant simulator, real time transient calculations

\section{INTRODUCTION}

Since 20 years, the National Centre for Scientific Research (CNRS, Grenoble-France) with French and European partners has focused R\&D efforts on the development of a new molten salt reactor concept called 
Molten Salt Fast Reactor (MSFR) selected by the Generation-IV International Forum (GIF) due to its promising design and safety features. The 'reference MSFR' design is a $3000 \mathrm{MW}_{\text {th }}$ reactor with a fuel salt volume of $18 \mathrm{~m}^{3}$, operated at a mean fuel salt temperature of $700^{\circ} \mathrm{C}$ [1]. R\&D activities are currently underway to ascertain whether MSFR systems can satisfy the goals of Generation-IV reactors in terms of sustainability, resource saving (closed fuel cycle, no uranium enrichment) and waste management (actinide burner), non-proliferation (integrated fuel cycle, multi-recycling of actinides), economics and safety. This has required the development of specific simulation tools to study such reactors with a liquid circulating fuel [2] acting also as coolant.

Molten salt reactors as liquid-fuelled reactors are indeed flexible in terms of operation (load-following capabilities...) or design (core geometry, fuel composition, specific power level...) choices, but they are very different in terms of design, operation and safety approach compared to solid-fuelled reactors. Such reactors call for a new definition of their operating procedures and safety approach. Dedicated developments and studies have been performed in the frame of the European SAMOFAR project of Horizon2020 and in parallel in France involving CNRS, CORYS and Framatome to develop a system code called LiCore adapted to such reactors, corresponding to a basic-principle power plant simulator.

The MSFR system includes three different circuits: the fuel circuit, the intermediate circuit and the power conversion circuit. The fuel circuit, defined as the circuit containing the fuel salt during power generation, includes the core cavity and the recirculation/cooling loops or sectors comprising notably the inlet and outlet pipes, pumps and fuel heat exchangers. The circulation period of the fuel salt on the fuel circuit s of 4 seconds during full power production. The neutronic model LiCore, at the centre of the simulator, corresponds to an improved point-kinetics model to take into account the specificities of a MSR, notably the circulation of the delayed neutron precursors out of the core. Coupled to a simple piston model for the fuel motion in the core, this code can perform calculations faster than real time to simulate the behaviour of the fuel circuit. The code can be run alone with a simplified model of the intermediate circuits to perform systematic transient studies of the MSFR fuel circuit during normal, incidental and accidental situations. Benchmarking of the LiCore code by comparison to the 3D coupled neutronic thermal hydraulics TFMOpenFOAM code have been done.

The LiCore code has also recently been integrated in the ALICES platform, the integrated simulation toolset designed by CORYS for the development, maintenance and operation of major simulator such as power plant simulators. The idea is to add a complete simulation of the intermediate and energy conversion circuits. This new version allows the study of the whole MSFR system, up to the definition of the operating procedures of the reactor. Some of the transient studies of the MSFR done by Framatome and CNRS using the LiCore code will be also detailed in the article.

\section{LiCore MODELING}

The fuel circuit and a simplified intermediate circuit are modelled in the LiCore code. This section describes the physics models implemented in the code.

\subsection{Specificities of Liquid Fuel Reactors}

Usual calculation codes for solid fuel reactors are not generic enough and thus improper for the simulation of liquid fuel reactors because of the strong coupling between neutronics and thermal hydraulics that occurs in the latter. In particular the circulation of the fuel salt causes the decay of some delayed neutrons precursors out of the active zone of the core, in a place of lower neutronic importance. When a liquid fuelled molten salt reactor such as the MSFR is critical, its reactivity $\rho$ is not null but slightly positive, accounting for the loss of delayed neutrons that would have participated in the chain reaction. It is therefore more natural to follow the margin to prompt criticality $\rho-\beta$, which at equilibrium equals the fraction of delayed neutrons that effectively take part in the chain reaction. This value depends on the circulation velocity of the fuel salt. 


\subsection{Neutronics Modelling: Point Kinetics per Zone}

The LiCore code [3] is based on a point-kinetics approach enhanced with a tracking of the delayed neutrons precursors in every mesh of the fuel circuit. When a delayed neutron is emitted, it is weighted according to the local neutronic importance of its emission zone. In this implementation, the adjoint flux is approximated as the neutron flux considered as combination of a Bessel function radially and a cosine vertically. The corresponding equations (1) and (2) are solved with an adaptive time step over the whole fuel circuit, for eight families of delayed neutrons precursors [2].

$$
\begin{gathered}
\frac{d n(t)}{d t}=\frac{\rho(t)-\beta}{\Lambda_{e f f}} n(t)+\sum_{f} \frac{\int \widehat{\phi(\boldsymbol{r})} \lambda_{f} P_{f}(\boldsymbol{r}, t) d \boldsymbol{r}}{\int \widehat{\phi(\boldsymbol{r})^{2}} d \boldsymbol{r}} \\
\frac{\partial P_{f}(\boldsymbol{r}, t)}{\partial t}+\boldsymbol{\nabla} \cdot\left(P_{f}(\boldsymbol{r}, t) \boldsymbol{v}\right)=\frac{\beta_{f}}{\Lambda_{e f f}} n(t) \hat{\phi}(\boldsymbol{r})-\lambda_{f} P_{f}(\boldsymbol{r}, t)
\end{gathered}
$$

Note that if the liquid fuel does not move, then the delayed neutron production $\lambda_{f} P_{f}(\boldsymbol{r}, t)$ will decay with the neutron flux distribution and then the weighting has no impact. If the liquid fuel is circulating, a part of the decaying precursors is located in low (adjoint) flux regions, and then the effective term of decaying precursors is smaller.

Kinetics parameters such as the generation time $\Lambda_{\text {eff }}$ of the fraction of delayed neutrons can be computed with a neutronic code such Serpent.

\subsection{Thermal Hydraulics Modelling}

A simplified one-dimension thermal hydraulics model is implemented. The circuits are divided into sections corresponding to a 1D pipe in which the fluid flows according to a mass flow rate imposed by the pump.

The meshing of the core region is slightly more refined in order to reproduce the velocity and power fields: it is divided into parallel sections which are themselves cut into smaller meshes. The flow distribution in the core is calculated so that the temperature at the top of the core region is homogeneous. This distribution can be optimized in the future in order to get a flow distribution closer to a CFD reference value.

The user can choose the boundary condition to be either the power extracted or the intermediate salt temperature in the heat exchanger located between the intermediate. He can also choose to not simulate the intermediate circuit and in that case the boundary condition is set in the primary heat exchanger.

If the simulation of the intermediate circuit is enabled or if the boundary condition is a fixed salt temperature, the power extracted from the fuel salt in the primary heat exchanger is computed thanks to the difference of temperature between the two salts. A volumetric heat exchange coefficient is deduced from correlations valid with a turbulent salt flow in a corrugated heat exchanger (in the current model).

In order to simplify the model, thermal diffusion has been neglected which is a valid approximation in normal operation of the MSFR, thermal convection being predominant over thermal conduction. Heat transfer due to turbulence is not modelled (except through the multi 1D mixing in the core), leading to a non-absorption of thermal fluctuations. Natural convection has not been implemented in the model at this stage which may limit the study of specific transients in which the primary mass flow decreases such as the coast down of the pumps.

\subsection{Steady-State Computation}

Every simulation made with LiCore starts with an initial steady-state computation. The power is fixed by the user. The fuel salt mean temperature is fixed at a reference temperature, the criticality temperature, 
which is iteratively adjusted to account for the neutron loss due to the delayed neutrons decaying out of the core. Finally the total amount of precursors of each family is fixed by the code by forcing the equilibrium between the decays and the productions. The intermediate salt temperature is also fixed iteratively in order to extract all the produced power from the fuel salt.

\section{TRANSIENT STUDIES WITH LiCore}

The LiCore code is designed to study the MSFR behaviour over time after a perturbation. The user has to define a scenario by giving the value of parameters such as inserted reactivity, power extraction or intermediate salt temperature and mass flow rate as a function of time. It is also possible to make parametric studies by giving more than one argument for a parameter. This section is an example of the results that can be obtained on two kind of transients: normal operation (load following) and accidental (reactivity insertions) transients.

\subsection{Load Following}

The first example of a transient study with the LiCore code corresponds to a load following. A parametric study is realized by modifying the extracted power in the heat exchanger located between the intermediate circuit and the electrical production circuit from 1.5 to $3 \mathrm{GW}$ with a period varying from 0 (instantaneous, not relalistic) to 100 seconds. Results are shown in Figure 1.
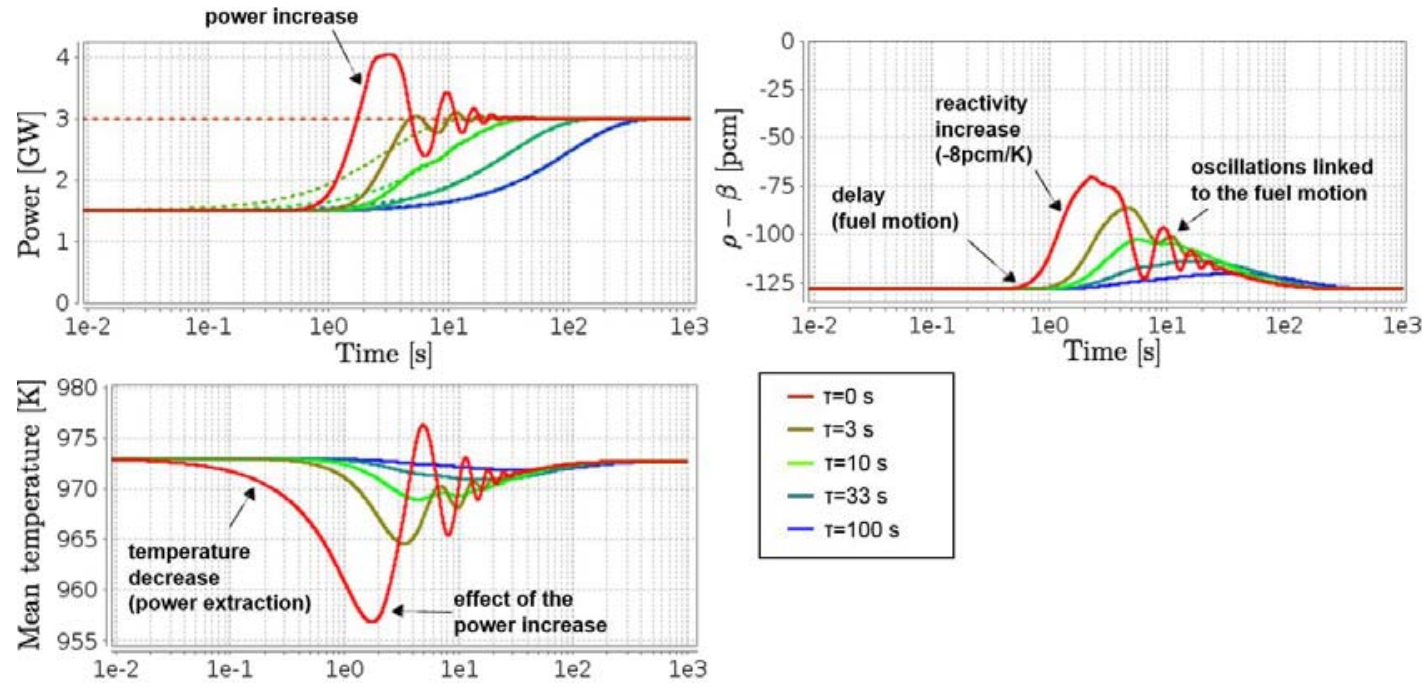

Figure 1. Load following transients from 1.5 to $3 \mathrm{GW}$ simulated with the LiCore code by varying the power extracted in the heat exchangers

The same pattern can be observed on the different curves. The heat extraction increase in the heat exchanger drives the mean temperature down and, after a small delay $(\sim \mathrm{s})$ due to the heat transport, the neutronics feedback increases the reactivity. Due to the reactivity increase, the neutronic power (plain line) increases and thanks to the good feedback coefficient, these curves follow the heat extraction (dashed lines) with a very good agreement if the time constant is around or larger than 10 seconds. We can see that, even if this is not realistic with an instantaneous modification of the heat extraction, the physical behaviour of the core is well simulated: some oscillations appear and the neutronic power reaches $4 \mathrm{GW}$ before stabilising because of a too large reactivity increases. 


\subsection{Reactivity Insertion}

Another transient study is shown here with the simulation of a reactivity insertion of $1000 \mathrm{pcm}$ in 1 second according to a linear law. The extracted power in the secondary heat exchanger is set to $3 \mathrm{GW}$. Figure 2 plots the results obtained with the LiCore code.

On a very short time scale, the reactivity increases sharply because of the insertion. It results in an increase of power and fuel salt temperature. The temperature feedback appears at around $0.1 \mathrm{~s}$ and perfectly compensates the reactivity insertion rate. After some seconds, the insertion ceases and the reactivity drops. The average fuel salt circulation time in the fuel circuit is of around 4 seconds during normal operation. Then between 1.5 to $3 \mathrm{~s}$, cold fuel salt arrives from the heat exchangers through the cold leg of the fuel circuit, enters the core cavity and replaces the fuel salt more heated by the reactivity insertion. This causes a jump of reactivity that recedes as the salt heated by the reactivity insertion returns back in the core after one turn through the cooling sectors. The reactor progressively settles into a new equilibrium at a higher temperature to balance the inserted reactivity, and the extracted power in the primary heat exchanger returns to its initial value of $3 \mathrm{GW}$ as the temperature difference between the salt of the fuel and intermediate circuits decreases.
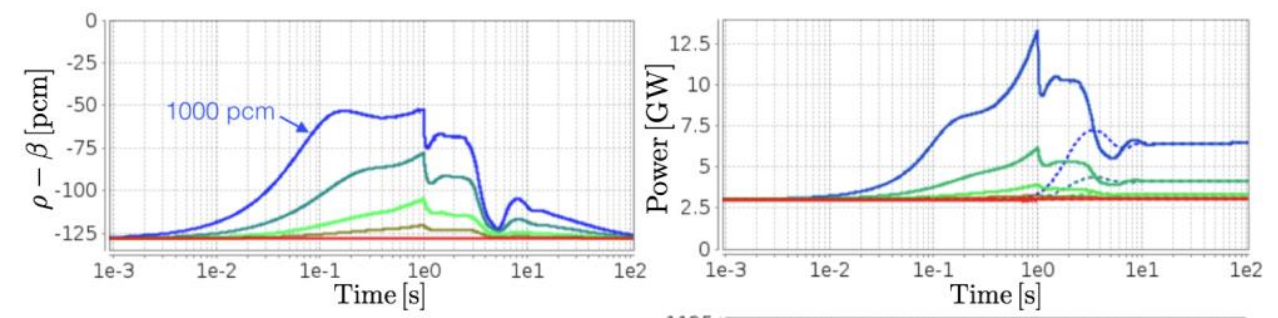

$$
\begin{array}{|l|}
\hline-0 \mathrm{pcm} \\
-33 \mathrm{pcm} \\
-100 \mathrm{pcm} \\
-333 \mathrm{pcm} \\
-1000 \mathrm{pcm} \\
\hline
\end{array}
$$

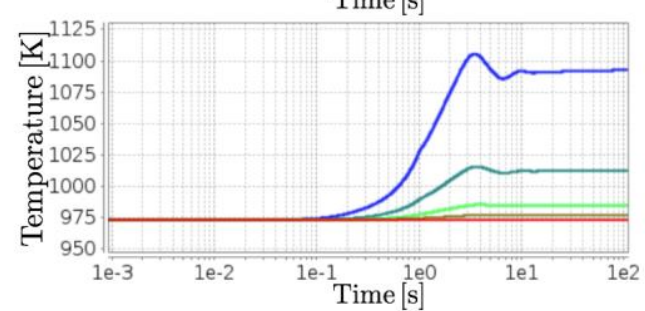

Figure 2. Transients of reactivity insertion in one second simulated with LiCore.

\section{COMPARISON OF THE LiCore CODE WITH OTHER CODES}

To be considered a reliable tool for safety, the code LiCore should undergo a verification and validation process. Comparisons have been led firstly to a point-kinetic model to check the neutronic model of LiCore (section 4.1) and to the 3D coupled neutronic-thermal hydraulic code TFM-OpenFOAM [2] (section 4.2).

\subsection{Comparison to a point-kinetic model}

Since the LiCore code was developed initially for the definition and the systematic study of normal operation transients of the MSFR, even if it appears to be able to correctly calculate also accidental transients, we chose here to compare LiCore to a point-kinetic model for a load-following transient. The point kinetic model developed in [4] is based on a modification of the fraction of delayed neutrons according to the amount decaying out of the reactor. This weighting is only valid at equilibrium with no modification of the flow rate as visible on the results detailed below.

To check the neutronic content of the LiCore code, a comparison has been performed with this classic point kinetics model on an instantaneous variation of the extracted power. The results are presented in Figure 3. The global behaviour is identical for both codes. We can observe that the LiCore model reproduces more precisely the core behaviour through oscillations physically explained by the fuel salt circulation, due to 
the variation of temperature and of precursor abundance in the salt exiting and re-entering the core after a short interval (circulation time of $\sim 4 s$ ).
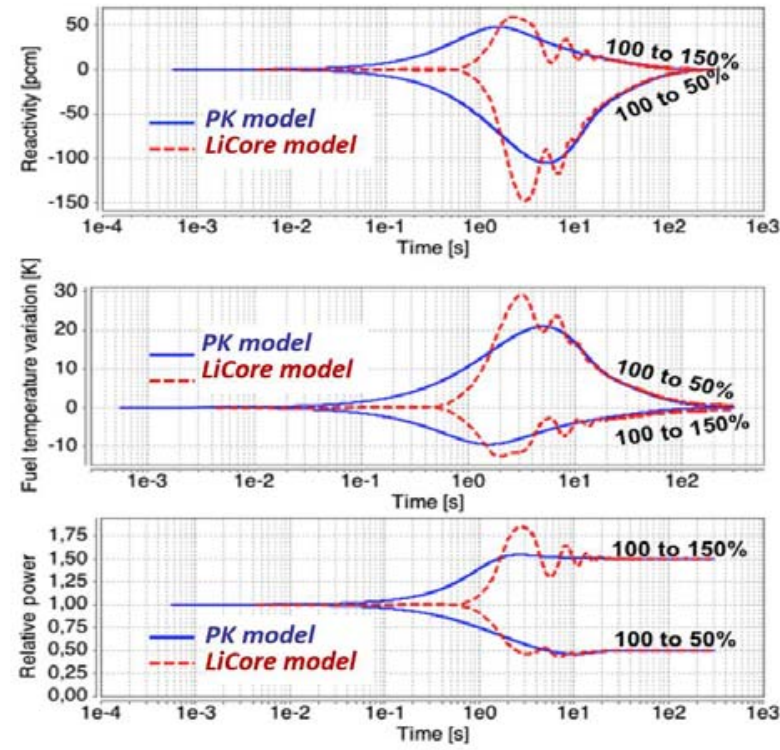

Figure 3. Comparison of LiCore and a point-kinetic model (PK).

The agreement between the results calculated by the two codes is globally satisfying, especially in the very beginning of the transient that depends mostly on the neutronics of the core. Note that, for a slower transient as expected for normal operation (i.e. load following in several minutes), the agreement is very good.

\subsection{Comparison to the TFM-OpenFOAM coupled code}

A comparison is detailed here on a transient of reactivity insertion of $1000 \mathrm{pcm}$ in 0.1 second to evaluate the stability of LiCore in a boundary case of accidental transient. The boundary condition set in LiCore has to match the one given in TFM-OpenFOAM with equivalent heat exchange coefficient, heat exchanger volume and intermediate temperature. Figure 4 plots the results obtained with the two codes, including also the calculation by the point-kinetic (PK) model mentioned in the previous section.
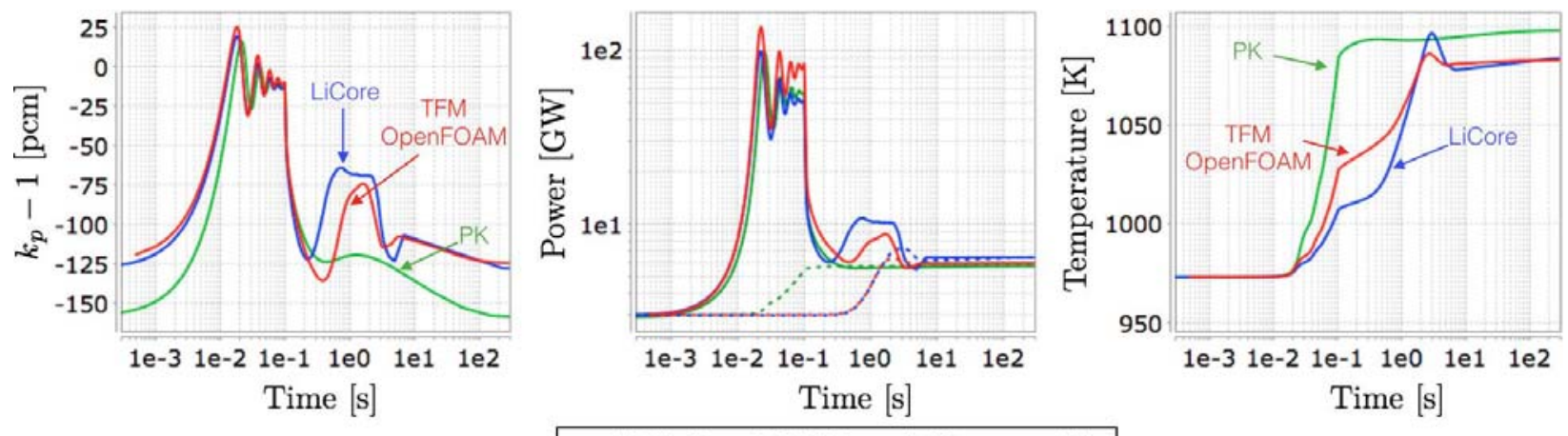

Figure 4. Comparison of LiCore, TFM-OpenFOAM and PK model on an insertion of $1000 \mathrm{pcm}$ in $0.1 \mathrm{~s}$.

The agreement between the results calculated by the two codes is globally satisfying, especially in the very beginning of the transient that depends mostly on the neutronics of the core. In particular, the agreement between the initial margin to prompt criticality is in the order of magnitude of a few pcm between TFM- 
OpenFOAM and LiCore. A discrepancy is observed between $0.5 \mathrm{~s}$ and $10 \mathrm{~s}$, due to the difference on the modelling of the fuel salt flow. Indeed the movement of the salt in LiCore is very simplified, and has trouble to represent accurately the impact of the entrance in the core of a colder or hotter volume of salt. However the order of magnitude is correct, and we can see that, compared to the classic PK model, the oscillation due to the fuel circulation at 1-3 seconds is correctly reproduced. One has to notice that the mean fuel temperature behaviour is well predicted (see right part of Figure 4).

\section{INTEGRATION OF THE LiCore CODE INTO THE MSFR SIMULATOR ALICES}

CORYS is providing an integrated simulation toolset, the ALICES platform, allowing users to build their own dynamic simulator. Building a simulator is made possible by designing elementary components integrating physics, assembling these components into circuits and running simulation with several circuits. Thanks to ALICES, any circuit can be modelled by connecting together elementary standard components (mechanic, thermo-hydraulic, electric, automation and human machine interface). Depending on the needs, OD or 1D modelling is available. ALICES is designed to build either basic principles nuclear power plant simulators or full scope simulators used for operators training and engineering studies.

CORYS brought its expertise to build a complete basic-principle MSFR simulator, based on ALICES standard libraries and the LiCore code for the fuel circuit modelling, in collaboration with the LPSC. The intermediate salt has been modelled as a monophasic fluid loop. The energy conversion circuit consists of a super-critical secondary loop, containing a turbine driving an alternator generating electricity.

In addition, basic instrument and control (I\&C) has been integrated to ensure main physical quantity remain steady in operation. As the secondary loop must remain super-critical in operation, its pressure and temperature need to be kept above the critical point. When the plant is not coupled to the grid, the turbine speed is controlled by limiting the amount of fluid sent to the turbine, whereas when the plant is coupled to the grid, the mechanical torque applied to the turbine needs to be controlled to stabilize the amount of electricity produced.

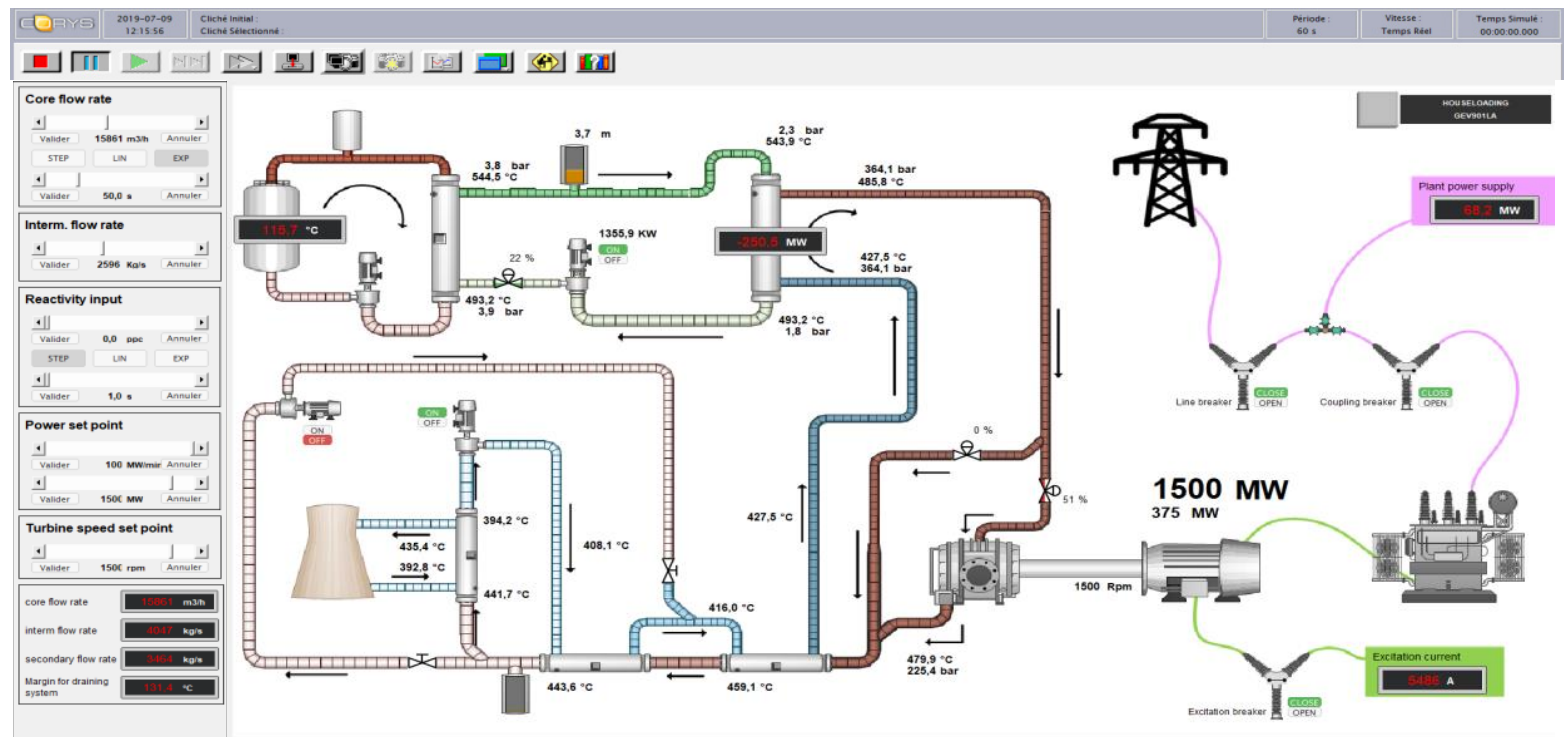

Figure 4. MSFR dynamic simulator general view.

Finally, a general operating interface has been created allowing the user to control main characteristic physical quantity or equipment of the MSFR, directly linked to the LiCore code (reactivity insertion, core pump speed), or with the energy conversion circuits (pump speed, pump state, electrical set point, grid connection breaker opening, ...). 
This simulator setup allows for the first time to study the behaviour of a MSFR core during transients such as load variations or house load operation.

\section{CONCLUSIONS AND PERSPECTIVES}

The LiCore code is now available either as in a standalone version allowing parametric studies or integrated in the ALICES platform as power plant simulator. The code will be used for the definition of the MSFR operation procedures in the frame of the SAMOSAFER European program and it will also be adapted to simulate a small modular version of the MSFR that be studied in the coming years. Finally, further developments will be done on LiCore itself (addition of the natural convection for example) and on its integrated version in ALICES (modelling of the components of the energy conversion circuit, improvement of the system regulations...).

\section{NOMENCLATURE}

$n(t)$ : time dependent component of the neutron population

$\mathrm{P}_{\mathrm{f}}(\mathbf{r}, \mathrm{t})$ : spatial distribution of family $\mathrm{f}$ of delayed neutron precursors

$\rho(t)$ : reactivity

$\beta$ : physical fraction of delayed neutrons

$\Lambda_{\text {eff: }}$ generation time

$\phi^{*}(\mathrm{E}, \mathbf{r})$ : adjoint neutron flux

$\chi(\mathrm{E})$ : prompt neutrons emission spectrum

$\chi_{\mathrm{d}, \mathrm{f}}(\mathrm{E})$ : delayed neutron emission spectrum

$\frac{\lambda_{f} P_{f}}{r}(\boldsymbol{r}, t)$ : normalized distribution of the delayed neutron emissions in the fuel circuit

$\widehat{v \Sigma_{f} \phi}(\boldsymbol{r})$ : normalized distribution of the prompt neutron emissions in the fuel circuit

$v$ : fluid velocity

$\widehat{\phi}(\boldsymbol{r})$ : normalized neutron flux

\section{ACKNOWLEDGMENTS}

The authors wish to thank the NEEDS (Nucléaire : Energie, Environnement, Déchets, Société) French Interdisciplinary program and the IN2P3 department of the National Centre for Scientific Research (CNRS) and Grenoble Institute of Technology for their support. This project has received funding from the EURATOM research and training programme 2015-2018 under grant agreement No 661891.

\section{REFERENCES}

1. M. Allibert, M. Aufiero, M. Brovchenko, S. Delpech, V. Ghetta, D. Heuer, A. Laureau, E. Merle-Lucotte, "Chapter 7 - Molten Salt Fast Reactors", Handbook of Generation IV Nuclear Reactors, Woodhead Publishing Series in Energy (2015).

2. A. Laureau et al., "Transient coupled calculations of the Molten Salt Fast Reactor using the Transient Fission Matrix approach", Nuclear Engineering and Design, 316, pp. 112-124 (2017).

3. A. Laureau, "Développement de modèles neutroniques pour le couplage thermohydraulique du MSFR et le calcul de paramètres cinétiques effectifs", PhD Thesis, Grenoble Alpes University, France (2015).

4. D. Lecarpentier, "Le concept AMSTER, aspects physiques et sûreté", PhD Thesis, EDF and CNAM, Paris, France (2001). 